\title{
Zweimal ein Versuch, den Überblick zu gewinnen
}

\section{Anna Sax}

lic. oec. publ., MHA, Mitglied der Redaktion
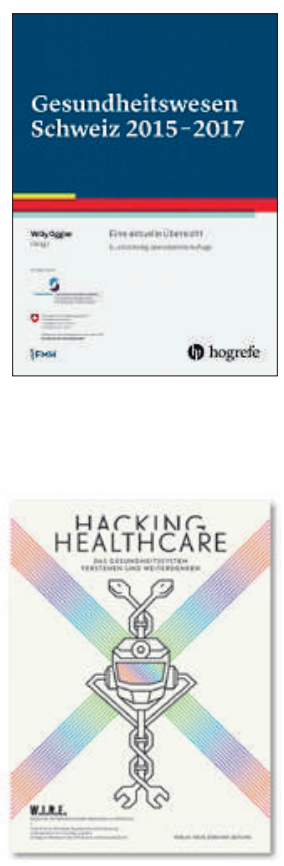

\footnotetext{
* Dazu gibt es auf der folgenden Seite ein Kurzinterview mit W. Oggier.
}

anna.sax[at]saez.ch
Willy Oggier (Hrsg.)

Gesundheitswesen Schweiz 2015-2017

Eine aktuelle Übersicht

Bern: Hogrefe Verlag; 2015.

488 Seiten. 39.90 CHF.

ISBN 978-3-456-85441-0

W.I.R.E. Thinktank für Wirtschaft, Gesellschaft und Life Sciences (Hrsg.)

\section{Hacking Healthcare}

Das Gesundheitssystem verstehen und weiterdenken Zürich: Verlag Neue Zürcher Zeitung; 2015.

468 Seiten. 60.00 CHF.

ISBN 978-3-03823-655-9

Die Intransparenz und der fragmentarische Aufbau des schweizerischen Gesundheitswesens bringt manche Leute zur Verzweiflung. Je genauer man hinschaut, desto komplexer wird es. Wo immer jemand an einer Schraube dreht, entsteht woanders eine neue Baustelle. Die Krankenkassenprämien steigen währenddessen kontinuierlich und schwächen die Kaufkraft der Haushalte. So erstaunt es nicht, dass das Bedürfnis aufkommt, die Komplexität in den Griff zu bekommen, zu verstehen, Lösungen zu formulieren. Dabei sind unterschiedliche Strategien möglich. Zwei Beispiele: Man trägt die Themen zusammen, die mit Gesundheitswesen zu tun haben, lässt Sachverständige darüber schreiben und ordnet sie alphabetisch zwischen zwei Buchdeckeln. Oder man sucht einen "ganzheitlichen Ansatz», der ebenfalls in ein Buch mündet.

Zwei Bücher sind im Herbst erschienen, die den Anspruch erheben, einen Überblick über das Gesundheitssystem der Schweiz zu vermitteln. Beim ersten Buch handelt es sich um die fünfte, vollständig überarbeitete Auflage des Klassikers Gesundheitswesen Schweiz.* Nach dem altersbedingten Rückzug des Mitherausgebers Gerhard Kocher ist nun der Gesundheitsökonom Willy Oggier alleiniger Herausgeber für die Ausgabe 2015-2017. Es ist ihm wieder gelungen, kompetente Autorinnen und Autoren für die 39 Kapitel zu finden. Der umfangreiche Wälzer enthält eine Fülle von Datenmaterial und eignet sich als Nachschlagewerk für Politikerinnen, Studierende, Praktiker, Journalistinnen und
Dozenten. Wie in den vorangegangenen Ausgaben treten auch Lobbyisten in Erscheinung, so sind etwa die Sponsoren FMH, santésuisse und BAG jeweils mit mehreren Autorinnen vertreten. Die Vielfalt von Themen und die Konzentration auf Fakten lässt jedoch wenig Spielraum für das Platzieren von Partikularinteressen. Speziell erwähnt sei das Kapitel über Gesundheitspolitik, das einen «Überblick im Überblick» gewährt und die Mechanismen der politischen Entscheidungsprozesse gut verständlich darstellt. Obwohl man sich fragen kann, ob eine alphabetische Anordnung der Themen Sinn macht, überzeugt die Methode, die im zweiten Buch gewählt wurde, noch weniger. Unter dem Titel Hacking Healthcare werden mit komplizierten Grafiken viele Binsenwahrheiten über das Gesundheitssystem vermittelt. Die Leserin erfährt, dass die Bevölkerung altert, der Pflegebedarf in Zukunft steigen wird und die Steuerung des ganzen Systems komplex ist. Hinzu kommen einige Zahlen und Fakten zur Pharma- und Medizintechnikindustrie. Der Thinktank W.I.R.E. als Herausgeber diskutierte über vier Jahre hinweg «hinter geschlossenen Türen sachlich und unvoreingenommen» mit Experten. Das Resultat wird im zweiten Teil in Form sogenannter Ideenkonzepte (u.a. "persönliche Gesundheitsdatenkonten», «Wikipedia-ähnlicher Reiseführer» oder «Hightech für Senioren»), vorgestellt. Diese namentlich gezeichneten Beiträge bilden den interessanteren Teil des Buches, sind jedoch in winzig kleiner Schrift und mit schlechtem Kontrast gestaltet, so dass das Lesen auch für gute Augen zu einer Herausforderung wird. Das Gleiche gilt für die Grafiken, die ein zentrales Element der ersten sechs Kapitel bilden. Das umfangreiche und aufwendig gestaltete Werk wird seinem Anspruch, das Verstehen und Weiterdenken zu fördern, nicht gerecht. Das Konzept bleibt unklar, das «Denkmodell» erschliesst sich nicht ohne weiteres.

Das Konzept von Gesundheitswesen Schweiz 2015-2017 ist einfach und klar. Der Nutzen besteht darin, dass man Informationen zu bestimmten Teilgebieten des Gesundheitssystems rasch nachschlagen kann. Dem erklärten Anspruch, einen Überblick über das Gesundheitssystem $\mathrm{zu}$ verschaffen, wird dieses Buch besser gerecht als Hacking Healthcare, das einen etwas ratlos zurücklässt. Letzteres kann zumindest für sich in Anspruch nehmen, nicht nur die Gegenwart abzubilden, sondern auch einen Blick in die Zukunft zu wagen. 


\section{Ein Beitrag zur begründeten Entscheidungsfindung}

\section{Herr Oggier, was ist der Anspruch Ihres Buches?}

Das Buch soll einen Überblick über das schweizerische Gesundheitswesen geben. In 39 Kapiteln schreiben 61 Autorinnen und Autoren über unterschiedliche Facetten des Systems. Das Buch beginnt mit dem Kapitel «Apotheken und Drogerien» und endet mit jenem über «Zahnmedizin».

\section{Will es vor allem eine Analyse bieten oder auch} konkrete Lösungsvorschläge für bestehende Probleme im Schweizer Gesundheitswesen?

Das Buch ist in erster Linie als Nachschlagewerk und Einführungslektüre gedacht. So hat beispielsweise der ehemalige Gesundheitsdirektor des Kantons Luzern und Präsident der kantonalen Gesundheitsdirektorenkonferenz Markus Dürr über eine frühere Ausgabe gesagt, dass er diese als Vorbereitungslektüre für seine damalige Departements-Übernahme las.

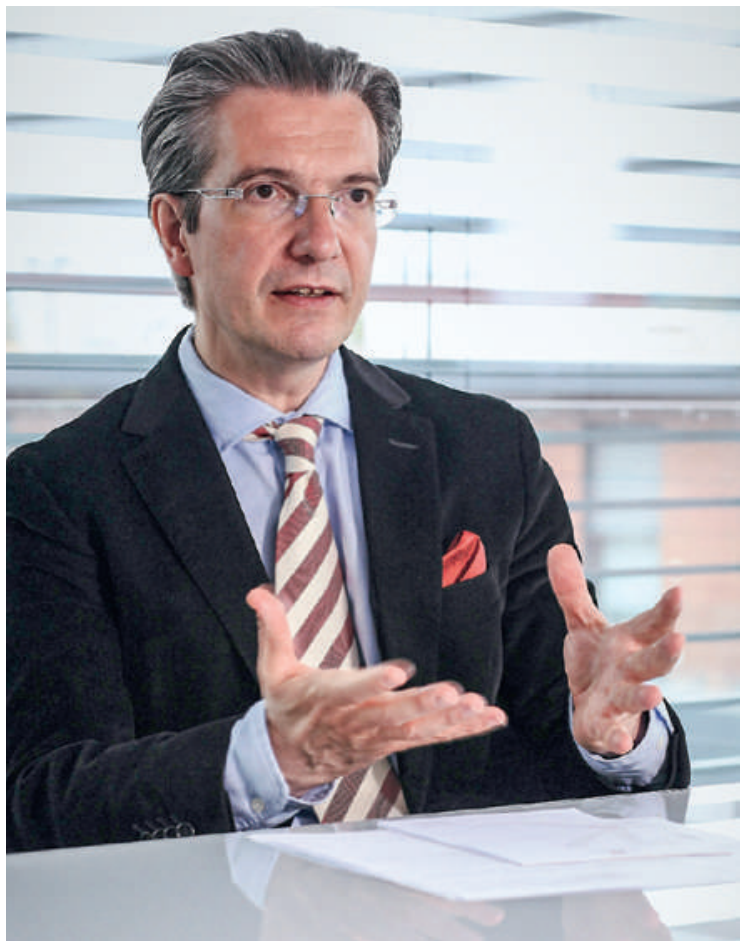

Willy Oggier, Herausgeber der neuen Auflage von Gesundheitswesen Schweiz 2015-2017.
Das Buch wird auch in sehr vielen Aus-, Weiter- und Fortbildungs-Veranstaltungen bei Ärzteschaft, Pflegenden, Therapeuten und Spitaldirektoren eingesetzt. Bei der letzten Ausgabe wurde festgestellt, dass sich auch deutlich mehr Bücher ins Ausland verkaufen liessen. Dieses Interesse hält auch bei der 5 . Auflage an. So hat etwa die Österreichische Ärztezeitung das Buch bereits zitiert und eine erste Anfrage einer deutschen Fachzeitschrift für eine Rezension liegt ebenfalls schon vor.

\section{Inwiefern hebt es sich von der vierten Auflage ab?}

Der bisherige Mit-Herausgeber Gerhard Kocher hat sich aus Altersgründen entschlossen, nicht mehr bei der Erstellung der 5. Auflage mitzuwirken. Anderseits hat erfreulicherweise neben den beiden bisherigen langjährigen grossen Trägern, dem Bundesamt für Gesundheit und dem Branchenverband der schweizerischen Krankenversicherer santésuisse, neu erstmals auch die FMH die Arbeiten finanziell mitunterstützt. Dieses Engagement ermöglichte u.a. erneut eine französische Übersetzung des Buchs. Dafür möchte ich mich an dieser Stelle bei allen drei Trägern bestens bedanken. Selbstverständlich hat die neue Ausgabe auch inhaltliche Erneuerungen erfahren. Zudem konnten teilweise neue Autoren für bisherige Artikel gewonnen, einzelne Kapitel weggelassen und neue hinzugefügt werden. $\mathrm{Zu}$ letzteren gehört beispielsweise eines, das die gesundheitspolitischen Entscheidungsprozesse in der Schweiz erörtert. Dies ist nicht zuletzt auch dem Umstand geschuldet, dass wir unseren ausländischen Leserinnen und Lesern die teilweise erheblichen Unterschiede zu ihren eigenen Systemen näherbringen wollen.

\section{Was leistet Ihre aktuelle Übersicht über das Schweizer} Gesundheitswesen nicht?

Es liefert keine Patentrezepte zur Lösung der anstehenden Probleme. Diese gibt es wahrscheinlich auch gar nicht. Aber wenn es sie gäbe, könnte das Buch wohl einen Beitrag zu einer fundierteren Entscheidungsfindung leisten.

Die Fragen an Willy Oggier stellte Isabel Zwyssig. 